\title{
RESET
}

Recherches en sciences sociales sur Internet

$3 \mid 2014$

TIC, familles et célébrations

\section{Introduction. La famille et les TIC au cœur des épreuves et des célébrations}

Introduction. Family and ICT at the Heart of Trials and Celebrations

Manuel Boutet et Laurence Le Douarin

\section{(2) OpenEdition}

Édition électronique

URL : http://journals.openedition.org/reset/349

DOI : $10.4000 /$ reset.349

ISSN : 2264-6221

Éditeur

Association Recherches en sciences sociales sur Internet

Référence électronique

Manuel Boutet et Laurence Le Douarin, «Introduction. La famille et les TIC au cœur des épreuves et des célébrations », RESET [En ligne], 3 | 2014, mis en ligne le 31 décembre 2014, consulté le 19 avril 2019. URL : http://journals.openedition.org/reset/349 ; DOI : 10.4000/reset.349

Ce document a été généré automatiquement le 19 avril 2019

(c) Association Recherches en sciences sociales sur Internet 


\section{Introduction. La famille et les TIC au cœur des épreuves et des célébrations}

Introduction. Family and ICT at the Heart of Trials and Celebrations

Manuel Boutet et Laurence Le Douarin

1 Ce numéro part du constat qu'aujourd'hui, l'édification des relations familiales s'appuie, notamment, sur l'utilisation de la téléphonie mobile et de la médiation électronique. Ainsi étudier les usages des outils de médiation électronique doit-il nous permettre d'en comprendre le rôle pour la famille, d'explorer par ce biais les normes familiales et d'élucider l'« économie morale » propre à chaque entité familiale (Silverstone et al., 1992). Les pratiques numériques peuvent servir $\mathrm{d}$ '« analyseurs pratiques » pour comprendre les dynamiques qui caractérisent cette institution (Le Douarin, 2007 ; Boutet, 2008). Car le numérique est devenu une infrastructure ordinaire de la vie quotidienne et notamment familiale: les parents et les enfants s'échangent des SMS pour s'informer de leurs activités (Martin, 2007) ; les couples règlent les affaires domestiques par le biais d'e-mails (Le Douarin, 2007) ; le téléphone ou les échanges électroniques servent de catalyseur pour se réunir autour des grands-parents et cultiver le lien familial (Segalen, 1999 ; Le Douarin \& Caradec, 2009), etc. De plus, l'avènement des derniers outils de communication rend possible l'occurrence du lien à tout instant et en tout lieu. Ils permettent également une surveillance continuelle tout en ouvrant de nouveaux espaces d'autonomie et d'intimité (Martin, 2003), par exemple dans le cas de l'infidélité conjugale (Le Douarin \& Le Van, 2010).

2 Pour tirer pleinement parti de cette fonction d'analyseur de l'entrée par le numérique, il nous a paru important, sans perdre de vue le quotidien, de mettre l'accent sur les moments forts de cette institution qui est en pleine transformation, c'est-à-dire les épreuves qu'elle traverse, les célébrations qui la soutiennent, et la manière dont elle s'y trouve parfois fragilisée, parfois renouvelée. 
Célébrations, car l'institution familiale n'est pas fondée seulement sur le quotidien, mais aussi sur des fêtes, des rituels au moment des repas, des célébrations lors des rencontres, sur des cérémonies autour de cadeaux et, désormais, également à travers des rituels médiatisés par les «nouvelles » technologies. Internet et les TIC ${ }^{1}$ participent en effet eux aussi à prolonger la façade familiale par une image idéalisée (Carmagnat et al., 2004), à la façon de cette «fonction sociale » de la photographie décrite par Pierre Bourdieu bien avant l'avènement du numérique, lequel remarquait lui-même qu'elle prolongeait déjà la fonction des portraits peints (Bourdieu, 1965 ; Boutet, 2009).

Epreuves, car les adversités qu'affronte la famille sont autant d'occasions de recomposition et de moments où les ressorts pour faire face sont mis à nu. Le cours de l'existence est en effet scandé par des bouleversements et des défis à surmonter. Or ces épreuves se trouvent souvent relativement standardisées, tels des rites de passage institués. Ainsi en va-t-il par exemple du passage à l'âge adulte (Van de Velde, 2008), de l'accès au marché du travail, de la mise en couple, de l'arrivée d'un enfant ou des recompositions familiales (Le Gall, 2005). Ils activent des processus d'ajustement aux changements introduits par lesquels les personnes reprennent prise sur leur vie. Or, bien que structurellement produites, ces épreuves successives se déclinent différemment à l'échelle des individus, dans leurs manières de s'y mesurer et d'y répondre, en fonction de leurs capitaux sociaux et de leurs contextes d'actions (Martuccelli, 2006). Les épreuves de la vie sont autant de moments dans lesquels se jouent non seulement les places et les rôles qu'occupent les membres de la famille mais aussi l'individualité de chacun en son sein en tant qu'acteur de sa propre existence. Dans ces processus, les usages d'Internet, de la téléphonie mobile et des TIC sont susceptibles de jouer un rôle central. Ils sont mobilisés dans des pratiques de communication et de soutien mutuel entre les membres de la famille, offrent à ces derniers la possibilité de s'en affranchir en se créant d'autres espaces d'intimité, permettent de trouver d'autres supports à l'expression et à la construction de l'individu. Ces outils participent aux redéfinitions des modes de fonctionnement familial.

5 Cette approche de la famille à partir de la contribution des pratiques numériques aux grands moments familiaux nous semble particulièrement ajustée pour relever le défi que constitue aujourd'hui l'étude d'une institution familiale entre permanences et mutations, et au-delà pour nous donner les moyens d'aborder les familles contemporaines dans leurs diversités et leurs incertitudes.

\section{Étudier les familles contemporaines}

6 La famille forme sous certains aspects l'unité de base du fonctionnement social (Durkheim, 1888). Cependant, la notion de famille n'est pas dépourvue d'une certaine ambiguité (Segalen, 1993). Alors que les anthropologues appréhendent sous ce terme l'ensemble des rapports de parenté étendue, les sociologues tendent à désigner par «famille » la famille nucléaire (Déchaux, 1995) et à étudier, dans la famille étendue, les liens familiaux et les rapports entre générations. La famille nucléaire - nouant ensemble conjugalité, filiation et fratrie - est caractérisée par des relations de collaboration et de cohabitation (Kellerhals et al., 1984). Or, compte tenu de la diffusion des outils de communication électronique dans les ménages, étudier ces relations demande aujourd'hui d'examiner la place qu'y occupent ces outils (Bigot \& Croutte, 2013). Parce qu'ils relient, les outils communicationnels font partie du scénario de la vie quotidienne. 
C'est leur place dans les relations familiales que ce numéro de la revue RESET propose d'interroger.

7 Depuis les années soixante-dix, les nombreux travaux qui auscultent la famille témoignent d'une évolution paradoxale, entre permanence et renouvellement. D'un côté, la famille conserve une place privilégiée dans la vie des acteurs : au niveau de sa réalité tangible comme au niveau des valeurs qu'elle véhicule comme premier espace de socialisation. Loin d'être émiettée, elle constitue toujours le socle fondamental de la sociabilité des individus et l'entraide familiale demeure primordiale pour surmonter les adversités et les défis que les acteurs rencontrent au cours de leur existence (AttiasDonfut et al., 2002 ; Déchaux, 2007). Pour autant, la famille dans sa forme nucléaire connaît une fragilité nouvelle, dont les manifestations sont connues : baisse des mariages, augmentation des divorces, émergences de nouvelles formes familiales, pluralité des fonctionnements familiaux, etc. Parallèlement, elle serait considérée au sein d'enjeux de plus en plus « affectifs » et intimes (Shorter, 1977 ; De Singly, 1996 ; Bawin-Legros, 2003). Ainsi, les individus cherchent à s'y « réaliser » dans des relations où l'affection prime sur l'autorité (De Singly, 1993, 1996, 2000). Considérée désormais comme un espace pour la construction de soi (De Singly, 1993, 2000), la famille fait face à des injonctions nouvelles. Les apparentés doivent désormais inventer et élaborer leurs relations aux autres. La teneur et les formes du lien familial ne vont plus nécessairement de soi. Aussi, à travers la définition de ces relations familiales se jouent les frontières entre les espaces collectifs propres à la vie de famille et les territoires personnels de chacun de ses membres. La tension entre une famille régie par des logiques d'obligation morale envers autrui et une famille comme espace de fidélité à soi se décline, en contexte d'épreuve et de formalisation des projets de vie, de manière nouvelle puisque les acteurs doivent formuler des compromis dans lesquels ils parviennent à demeurer libres et maitres de leur vie personnelle, sans pour autant se désengager de la vie familiale (Martuccelli, 2006).

De ces transformations, certains auteurs soulignent combien la famille contemporaine est devenue "incertaine», faite de choix et de décisions individuels (Roussel, 1989), et marquée par la privatisation des liens. Et si d'autres nuancent la portée de l'affaiblissement des normes qui régulent l'institution, c'est pour témoigner de la diversité des ajustements familiaux (Kellerhals, 2004). Ainsi, l'être en famille est plus que jamais marqué par un impératif de communication et de négociation entre ses différents membres. Une injonction nouvelle qui coïncide avec une décohabitation de plus en plus récurrente en raison des recompositions familiales mais aussi la plus grande mobilité des individus, due notamment aux transformations du marché du travail et des parcours éducatifs.

\section{Épreuves et équipements symboliques}

9 Ce numéro de la revue RESET s'interroge donc sur la manière dont les outils informationnels et communicationnels sont des appuis ou des éléments de mobilisation dans les pratiques, les épreuves et les célébrations familiales, ainsi que sur les formes de composition et de recomposition des routines, relations ou hiérarchies familiales. Au final, comment les TIC sont-elles utilisées pour tisser du lien et faire famille, et que nous révèlent-elles des multiples tensions qui animent l'être en famille lors des étapes charnières de la vie, telles que le passage à l'âge adulte, la mise en couple, l'arrivée d'un 
enfant, sa scolarisation, la mobilité résidentielle, la séparation, la retraite ou la dépendance?

Les techniques numériques n'ont pas un rôle univoque au cours de l'histoire familiale d'où la nécessité d'enquêtes empiriques pour étudier quelles fonctions leurs sont dévolues et quels sont leurs « effets » relatifs. En effet, dans la diversité de leurs formes et de leurs pratiques, ces outils facilitent ou permettent aussi bien de consolider que de remettre en cause l'image et l'unité de la famille. D'un côté ils soutiennent des logiques d'obligations envers le groupe familial : les cérémonies ou les rituels familiaux tels que les "visites téléphoniques", les séances de webcam, tout ce qui renvoie à la «culture du lien », ou encore les albums photographiques et l'entretien "online » d'une mémoire partagée ; de l'autre côté ils équipent des logiques d'émancipation : intimité électronique, ubiquité, etc. Ils favorisent non seulement les relations intersubjectives au sein d'une alcôve électronique mais aussi des processus de publicisation des espaces de l'intime (Cardon \& Delaunay-Téterel, 2006).

11 Le dossier de ce numéro s'organise autour de deux moments que sont l'enfance et le grand-âge. Chacun des textes est centré autour d'une épreuve rencontrée dans l'un de ces moments, et sur la manière dont les techniques numériques interviennent dans la manière dont les acteurs en prennent conscience et y font face. Les épreuves sont chaque fois marquées par un dilemme moral : éduquer ses enfants à la culture scolaire tout en préservant leur épanouissement personnel, surveiller ses enfants tout en préservant leur autonomie, assister ses aînés tout en respectant leur individualité. Les réponses qui y sont apportées ne sont pas des décisions légères. Elles ne sont d'ailleurs jamais uniques, et font souvent l'objet du soin des individus et de l'attention des familles. Aussi appellent-elles en retour de la part du chercheur une enquête empirique attentive à l'expérience des enquêtés comme aux processus collectifs en jeu.

\section{Les épreuves d'éducation et le processus d'autonomisation}

12 Loin de faire l'expérience d'un affaiblissement des normes, les familles produisent des ajustements variés pour maintenir la cohésion et réguler les comportements. C'est cette diversité des ajustements familiaux que Marie-Christine Le Floch se propose d'étudier par l'intermédiaire d'Internet et d'autres TIC en plongeant le lecteur dans les différentes formes d'articulation entre les obligations scolaires - par les exercices à faire à la maison - et le temps libéré de l'école, entre le souci des parents de voir leur enfant réussir leur scolarité - et notamment les apprentissages de la lecture et de l'écriture - et le respect de son épanouissement personnel (De Singly, 1996), entre la culture des écrans et la culture de l'imprimé. Ainsi, l'auteure interroge l'éducation des enfants dans le rapport à l'école et le rôle des écrans (opposés aux supports imprimés) dans la sphère familiale, en consonance ou non avec le cadre scolaire. Elle adopte une position originale en repérant les tensions propres aux familles quand, après l'école, leurs enfants scolarisés au cours préparatoire ont des devoirs scolaires à réaliser de retour au domicile, lequel regorge de sollicitations potentiellement interruptives: télévision, console de jeu, ordinateur connecté. Entre "après l'effort, le réconfort ", «le réconfort avant l'effort » ou encore "l'effort dans le réconfort", les parents présentent des postures multiples et variées pour choisir le moment propice à la réalisation des devoirs. Si, en principes, il n'y a pas de 
devoirs écrits au CP, il reste qu'il y a «toujours quelque chose à faire » en continuité avec le cadre scolaire, ce que traduit la notion d'externalisation du travail scolaire » (Rayou, 2010). Le cours préparatoire ( $\mathrm{CP}$ ) constitue une étape décisive pour le démarrage de la lecture et l'entrée dans la maitrise de l'expression écrite, et les enfants - comme leurs parents - passent une épreuve remplie de tests et de verdicts sur l'acquisition des premiers apprentissages de la culture écrite. Le moment particulier du retour à la maison confronte deux mondes qui peuvent entrer en dissonance. Il y a celui des exigences scolaires pour franchir cette étape charnière du CP. Se superpose également celui d'un temps plus lâche, suspendu hors des murs de l'école, certes toujours contraint, mais avec des points de distorsion vis-à-vis de la « sous-traitance pédagogique » imposée par l'école aux parents. Marie-Christine Le Floch distingue alors les différentes manières des parents de se faire plus ou moins le relais de l'école. Selon les postures éducatives endossées par les parents (sérieux scolaire vs sérieux à l'école) et le type de contrôle qu'ils exercent sur les usages des écrans (surveillance vs guidage), quatre types de rapport à l'épreuve d'éducation au CP se dessinent : «l'ordre scolaire », « les deals », « les contrariétés », « le continuum et l'hybridation des supports». Cette typologie ne fait cependant pas l'économie d'une sociologie des usages des supports écrans et imprimés et rompt avec la dichotomie entre l'écran-futile et l'imprimé-légitime : les écrans peuvent incarner à la fois des fonctions oisives et pédagogiques, l'imprimé offrir à la fois une possibilité d'évasion et un support didactique (Poliak \& Mauger, 1998).

Clément Rivière interroge le processus d'autonomisation des enfants et l'expérience de la mobilité dans la ville. Il souligne combien le téléphone mobile pour les enfants - comme pour les adultes - constitue simultanément un instrument d'émancipation de la sphère familiale et de renforcement de la surveillance exercée par celle-ci. La famille est traversée par cette tension entre émancipation et contrôle, et l'auteur la perçoit d'autant mieux dans la régulation des déplacements et des activités des enfants dans les espaces publics urbains, ici à Paris et à Milan. Dans quelle mesure l'encadrement parental facilitet-il ou interdit-il l'équipement en téléphonie mobile des enfants ? Dans un premier temps, Clément Rivière détaille les raisons pour lesquelles certains parents équipent leur progéniture aux portes de l'adolescence : ne pas les exclure des formes que prennent la sociabilité juvénile, faciliter l'assouplissement des cadres temporels et gérer les imprévus (panne ou grève des transports, absence d'un enseignant, etc.), se rassurer en maintenant le fil à distance et en utilisant le téléphone mobile comme "signal d'alarme », réduire les appréhensions des enfants en décourageant les agresseurs potentiels. Dans un deuxième temps, l'auteur explore les significations du non-usage et les raisons pour lesquelles certains parents sont au contraire réticents à l'adoption du téléphone mobile et à l'équipement de leurs enfants. Les logiques de freinage sont nombreuses: résister à l'invasion de ces outils qui accélèrent le quotidien, refuser la tyrannie de la majorité imposée par les groupes de pairs au profit de la culture de la singularité, relativiser la fonction de réassurance que procurait le téléphone mobile, limiter les nouveaux risques liés à l'équipement des enfants en téléphonie mobile (accidents, agressions, vols) et enfin remettre en cause l'acquisition des compétences d'autonomie, le téléphone mobile étant perçu comme une « solution de facilité » qui nuirait à la capacité des enfants à gérer les situations sans l'aide des parents au bout du fil. Troisièmement, Clément Rivière tente d'expliquer le contraste des positionnements à l'égard de l'équipement du téléphone mobile en fonction des logiques éducatives des parents, de leur appartenance sociale et des conditions d'exercice du travail parental (monoparentalité, parents séparés). Les 
résultats renvoient à des logiques éducatives plus larges et socialement clivées: "l'acquisition valorisée en milieu populaire d'un instrument rassurant, qui fait par ailleurs plaisir à l'enfant et contribue à son intégration sociale s'oppose à des logiques de freinage plus caractéristiques des parents les plus diplômés, qui visent à favoriser l'acquisition de compétences de mobilité et la protection de l'intégrité physique de l'enfant ». La monoparentalité ou les parents séparés constituent deux cadres propices à l'adoption d'un téléphone mobile pour les enfants. Dans les familles monoparentales, le travail d'encadrement est plus solitaire et les parents dans cette situation familiale ne sont pas toujours disponibles pour accompagner les enfants dans leurs déplacements. Le téléphone mobile remplit alors une fonction de réassurance lors des déplacements urbains. Dans les familles séparées ou divorcées, équiper le lien filial par l'achat d'un téléphone mobile offre la possibilité de développer une relation plus personnalisée et plus individualisée avec l'enfant, que ce dernier réside en alternance ou non chez l'autre parent.

\section{Les relations intergénérationnelles à l'épreuve du vieillissement}

Lin étudie un rôle nouveau que les jeux vidéo viennent à jouer dans l'espace domestique à travers les cadeaux technologiques - notamment la console de jeu Wii offerts par les enfants à leurs parents âgés. L'auteur montre combien les enfants dans le processus d'acquisition sont soumis à des injonctions paradoxales : peut-on offrir un cadeau technologique à une population plutôt réfractaire à l'appropriation d'un dispositif qui ne semble pas « de leur âge »? Faut-il acheter une console de jeu au prix relativement élevé sans être assuré que les parents âgés en fassent usage ? L'achat d'un tel dispositif exprime-t-il un dévouement des descendants envers leurs ascendants pour les maintenir en forme plus longtemps (exercices physiques et cognitifs sur les jeux Wii) ou une sorte de délégation qui érige la console de jeu au statut de « babysitter » - comme peut l'être la télévision à domicile - et permettre ainsi aux descendants de vaquer à d'autres occupations ou d'être rassurés qu'en leur absence le temps des parents âgés reste rempli et non soumis à l'ennui ? offrir ce type de cadeau technologique tire-t-il un trait d'union entre les générations ou renforce-t-il les appartenances générationnelles?

16 A travers la construction de cette nouvelle pratique, l'auteur souligne combien la pratique des jeux vidéo prend au fil de son appropriation une épaisseur non-univoque. La console est à la fois à la mode et abordable, elle permet aux enfants de tenir leurs parents occupés en leur absence, et, pour les générations qui cohabitent, elle représente également un moyen de s'amuser tous ensemble de retour au domicile. Plus largement, la console reste une pratique qui soude les groupes de pairs des jeunes adultes, tout en 
devenant aussi une manière de combler le fossé générationnel avec ses parents. Lorsque les parents acceptent la console, cela peut être pour eux une manière d'apprendre de leurs enfants, en même temps que ceux-ci peuvent y voir parfois une forme de reconnaissance de leur mode de vie - par-delà certaines critiques subies au fil des années, formulées par leurs parents selon les normes propres à leur génération.

\section{Nouvelles représentations ou nouvelles familles?}

Le dernier article, proposé dans la rubrique Perspectives, adopte une approche issue des études cinématographiques et sémiologiques pour étudier les célébrations familiales. À travers l'exemple du mariage, il discute les transformations induites par les dispositifs techniques (caméras, formes de diffusion et de circulation des films) dans la capture de ces célébrations. Giuseppina Sapio y souligne qu'Internet et les TIC sont des équipements à la fois fonctionnels et symboliques - non seulement pour les contenus qu'ils véhiculent mais également pour les formats qu'ils permettent de fabriquer. À partir d'un petit corpus de films de mariage sur YouTube, l'auteure montre que le film de mariage prolonge l'album photographique dans l'ordre des commémorations des cérémonies familiales (Bourdieu, 1965). Mais elle montre aussi que les pratiques sociales de l'image n'ont plus aujourd'hui l'homogénéité qu'on leur connaissait avant le web. En suivant cette piste, elle suggère que le numérique participe de l'ouverture contemporaine des interprétations de la cérémonie du mariage - l'écriture filmique permet en effet aux membres de la famille de découper des séquences correspondant aux moments rituels et de fixer ainsi, pour discussion et pour mémoire, la reformulation des codes cérémoniels.

Pour cela, Giuseppina Sapio étudie la mise en scène des moments cérémoniels de la vie familiale dans les "homes movies» (ou "film[s] de famille réalisé[s] par et pour la famille») et leur évolution avec l'avènement du numérique. Internet et les TIC jouent aujourd'hui un rôle important dans les pratiques par lesquelles le groupe familial se présente aux protagonistes qui figurent à l'écran, à ses proches, à des amis, aux collègues, à des connaissances, voire à des inconnus par l'intermédiaire des plateformes électroniques. À cela s'ajoute que les « home movies » ne sont pas de simples captations, mais un élément important des commémorations que préparent les cérémonies, comme P. Bourdieu le remarquait avec la pratique de l'album photographique (Boutet, 2009). Après une tentative de définition des home movies au croisement entre la pratique amateur et une forme d'expression artistique dont la légitimité est controversée, l'auteur passe au crible les moyens utilisés pour s'adonner à cette pratique : Super 8, VHS, vidéos numériques. Elle montre combien la mise en scène des home movies ne renvoie pas seulement à la mémoire familiale mais aussi aux identités de chacun et à la reconnaissance mutuelle des rôles attribués dans les séquences filmées. Enfin, Giuseppina Sapio pointe des changements qu'introduit le numérique dans la pratique des home movies et notamment les nouvelles modalités de partage de contenus privés grâce aux réseaux sociaux de type YouTube ou Dailymotion. Les changements se retrouvent dans les conditions de tournage aussi bien que dans le choix des prises : la miniaturisation et la portabilité des caméscopes numériques favorisent la circulation parmi les membres du groupe, en multipliant les points de vue sur le même événement, alors que le Super 8 ratifiait plutôt une division des tâches où le père de famille prenait en main le dispositif technique sans céder facilement à d'autres membres de sa famille le port de la caméra et les prises de vue. En outre, la tendance au multi-équipement et à l'individualisation des 
supports favorise elle aussi la pluralité des points de vue et l'intervention de personnes multiples dans la réalisation des home movies. Enfin, le partage avec des inconnus sur des plateformes de type YouTube ouvre la voie aux commentaires et à des formes de reconnaissance sur les réseaux sociaux. Plus généralement, loin d'épouser un déterminisme sociotechnique qui renverrait aux dispositifs techniques la puissance de bouleverser le partage des rôles sociaux, l'auteure montre combien les évolutions de la famille et des rapports en son sein trouvent un écho dans l'appropriation de tel ou tel dispositif. C'est aussi parce que la famille a évolué qu'elle trouve dans la miniaturisation et l'individualisation des supports des éléments d'expression de ces différents portraits.

\section{BIBLIOGRAPHIE}

AtTIAS-DONFUt Claudine, LAPIERRE Nicole \& SEGALEN Martine (2002). Le nouvel esprit de famille, Paris, Odile Jacob.

BAWIN-LEGRos Bernadette (2003). Le nouvel ordre sentimental : à quoi sert la famille aujourd'hui ? Paris, Payot.

BIDET Alexandra (2007). « Le corps, le rythme et l'esthétique sociale chez André Leroi-Gourhan », Techniques et culture, 46-47, pp. 15-38.

Bigot Régis \& CROUTTE Patricia (2011). La diffusion des technologies de l'information et de la communication dans la société française, Credoc, octobre.

BOURDIEU Pierre (1965). Un art moyen. Étude sur les usages sociaux de la photographie, Paris, Les Éditions de Minuit.

Boutet Manuel (2009). « Un objet peut en cacher un autre. Relire Un art moyen de Pierre Bourdieu au regard de trente ans de travaux sur les usages », Réseaux, 155, pp. 180-214.

CARADEC Vincent (2007). «L'épreuve du grand âge », Retraite et société, 52, pp. 11-37.

CARDon Dominique \& Delaunay-Teterel Hélène (2006). « La production de soi comme technique relationnelle ", Réseaux, 138, pp. 15-71.

CARmagnat Fanny, Deville Julie \& Mardon Aurélia (2004). « Une vitrine idéalisante, les usages des sites familiaux », Réseaux, 123, pp. 175-203.

DeCHAUX Jean-Hugues (1995). « Orientations théoriques en sociologie de la famille : autour de cinq ouvrages récents ", Revue française de sociologie, 36(3), pp. 525-550.

DeChauX Jean-Hugues (2007). Sociologie de la famille. Paris, La Découverte.

DE SINGLY François (1993). Sociologie de la famille contemporaine, Paris, Armand Colin.

De SINGLY François (1996). Le soi, le couple et la famille, Paris, Nathan.

DE SINGLY François (2000). Libre ensemble. L'individualisme dans la vie commune, Paris, Nathan. 
DE SINGLY François (2003). « Intimité conjugale et intimité personnelle : à la recherche d'un équilibre entre deux exigences dans les sociétés modernes avancées ", Sociologie et sociétés, 35(2), pp. 79-96.

DuRкнеiм Émile (1888). «Introduction à la sociologie de la famille », In : Émile Durkheim, Textes. 3. Fonctions sociales et institutions, Paris, Les Éditions de Minuit, pp. 9- 34.

HARTMUt Rosa (2010), Accélération. Une critique sociale du Temps, Paris, La Découverte.

Illouz Eva (2006). Les sentiments du capitalisme, Paris, Seuil.

KaUfMANN Jean-Claude (1997). Le cœur à l'ouvrage. Théorie de l'action ménagère, Paris, Nathan.

Kellerhals Jean, Troubot Pierre-Yves \& LAzega Emmanuel (1984). Microsociologie de la famille, Paris, PUF.

KeLLERHALs Jean, WIDMER Eric \& LÉVY René (2004). Mesure et démesure du couple. Cohésion, crise et résilience dans la vie des couples, Paris, Payot.

LE DOUARIN Laurence (2007). Le couple, l'ordinateur, la famille, Paris, Payot.

LE DOUARIN Laurence \& CARADEC Vincent (2009). « Les grands-parents, leurs petits-enfants et les « nouvelles » technologies de communication », Dialogue, 186, pp. 25-35.

LE GALL Didier (2005). « La conception de l'habiter à l'épreuve de la recomposition familiale ", Espaces et sociétés, 120-121, pp. 45-60.

MARTIN Corinne (2007), Le téléphone portable et nous. En famille, entre amis, au travail, Paris, L'Harmattan.

MARTIN Corinne (2003). « Le téléphone portable : machine à communiquer du secret ou instrument de contrôle social ?", Communication et langages, 136, pp. 92-105.

MARTUCELLI Danilo (2006). Forgé par l'épreuve. L'individu dans la France contemporaine, Paris, Armand Colin.

MASSIT-FOLLEA Françoise (2004). « Research trends on Internet Uses: a French perspective », Conférence Internet, Culture, and Society : French and American Perspectives, 18-20 Novembre, Austin, Texas University.

POLIAK Claude F. \& MAUGER Gérard (1998). « Les usages sociaux de la lecture », Actes de la recherche en sciences sociales, 123, pp. 3-24.

MAUSs Marcel (1950). « Les techniques du corps », In : Sociologie et anthropologie, Paris, PUF/ Quadrige.

ROUSSEL Louis (1989). La famille incertaine, Paris, Odile Jacob.

SEGALEN Martine (1993). Sociologie de la famille, Paris, Armand Colin.

SHORTER Edward (1977). Naissance de la famille moderne, Paris, Seuil.

SILVERSTONE Roger, HIRSH Eric \& MORLEY David (1992). « Information and communication technologies and the moral economy in the house hold ", in Consuming Technologies, Media and Information in Domestic Spaces, London, Routledge.

THERY Irène (1998), Couple, filiation et parenté aujourd'hui, Paris, Editions Odile Jacob.

THEVENOT Laurent (1994). «Le régime de familiarité. Des choses en personne », Genèses, 17, pp. 72-101. 
VAN DE VELDE Cécile (2008). Devenir adulte. Sociologie comparée de la jeunesse en Europe, Paris, PUF.

\section{NOTES}

1. Technologies de l'information et de la communication.

\section{AUTEURS}

MANUEL BOUTET

Université de Nice Sophia-Antipolis, GREDEG

\section{LAURENCE LE DOUARIN}

Université de Lille 3, CeRIES 